\title{
ANALYTICAL STUDY OF COLLECTOR SOLAR-GAIN ENHANCEMENT BY MULTIPLE REFLECTORS
}

\author{
RAKESH KUMAR, S. C. KAUSHIK, $\dagger$ and H. P. GARG \\ Centre for Energy Studies, Indian Institute of Technology, Hauz Khas, New Delhi-110 016, India
}

(Received 6 May 1994; received for publication 6 December 1994)

\begin{abstract}
We have considered the general case of a collector with four reflectors. An analytical model is developed for study of the effect of an individual reflector on the collector. Reflectors R-1, R-2, R-3, and R-4 face south, north, west, and east, respectively. For a collector with two reflectors R-1 and R-2 on the southern and northern sides, up to $44 \%$ enhancement is achieved in the solar gain for December (winter) and up to $14 \%$ for May (summer). However, due to shadowing, two additional reflectors R-3 and R-4 on the eastern and western sides cause negligible additional solar gain. Without shadowing, enhancement in solar gain due to reflectors R-3 and R4 is $14-36 \%$ for May and $44-56 \%$ for December for the Delhi climate.
\end{abstract}

\section{INTRODUCTION}

A flat-plate collector is the simplest means for converting solar energy into useful heat. A flat-plate collector collects both direct and diffuse components of incident solar radiation. If the collector surface is normal to the incoming solar radiation, the heat gain is a maximum. In practice, it is not always possible to tilt the collector surface towards the sun. In such cases, higher concentrations of solar radiation on the collector are achieved by the addition of one or more reflectors surrounding the collector. A reflector does not only increase the collection area but also redirects the solar radiation closer to normal incidence.

There is published literature on collector-reflector combinations. Tabor ${ }^{1}$ studied the effect of a specular reflector on the collector by using a graphical method. McDaniel et $\mathrm{al}^{2}$ studied the effect of a planar reflector on the performance of a collector. Seital ${ }^{3}$ studied the effect of both diffuse and specular reflectors on the collector. Most of the published work deals with one reflector. ${ }^{4-7}$ Garg and Hrishikesan ${ }^{8}$ and Kaushik et $\mathrm{al}^{9}$ considered two reflectors, one facing the southern side and the other the northern side of the collector, and optimized the tilts of these reflectors for maximum solar gain.

We consider a collector with four reflectors, one on each side of the collector. An analytical model is developed to evaluate the optimum reflectors tilt for maximum output from the collector. When the collector faces south and has a $0^{\circ}$ tilt with respect to the horizontal, it is found that multiple reflectors can improve collector performance significantly.

\section{ANALYSIS}

The configuration of the collector-reflector system is shown in Fig. 1. The reflectors R-1, R-2, R3 , and R-4 are located at the four sides of the collector and are connected edge-to-edge to the collector. The tilts of the collector and reflectors are variable and the whole system has an off-south angle $\phi$. Assumptions made in the analysis are: (i) the reflectors are specular with reflectivity $\rho_{r}=0.8$. (ii) The collector surface is a blackened flat plate. (iii) The transmittance-absorptance products are functions of the angle of incidence. (iv) Shading by the collector and of reflectors on each other is neglected but shading of reflectors on the collector is considered. (v) Multiple reflection between reflectors is neglected. (vi) Addition of reflectors between the collector and sky does not affect the shape factor. (vii) Each month of the year is represented by an average day. Values of the constants used are given in Table 1. (viii) The analysis takes into account only absorption of solar radiation by the blackened plate.

The total energy absorbed by the collector is 


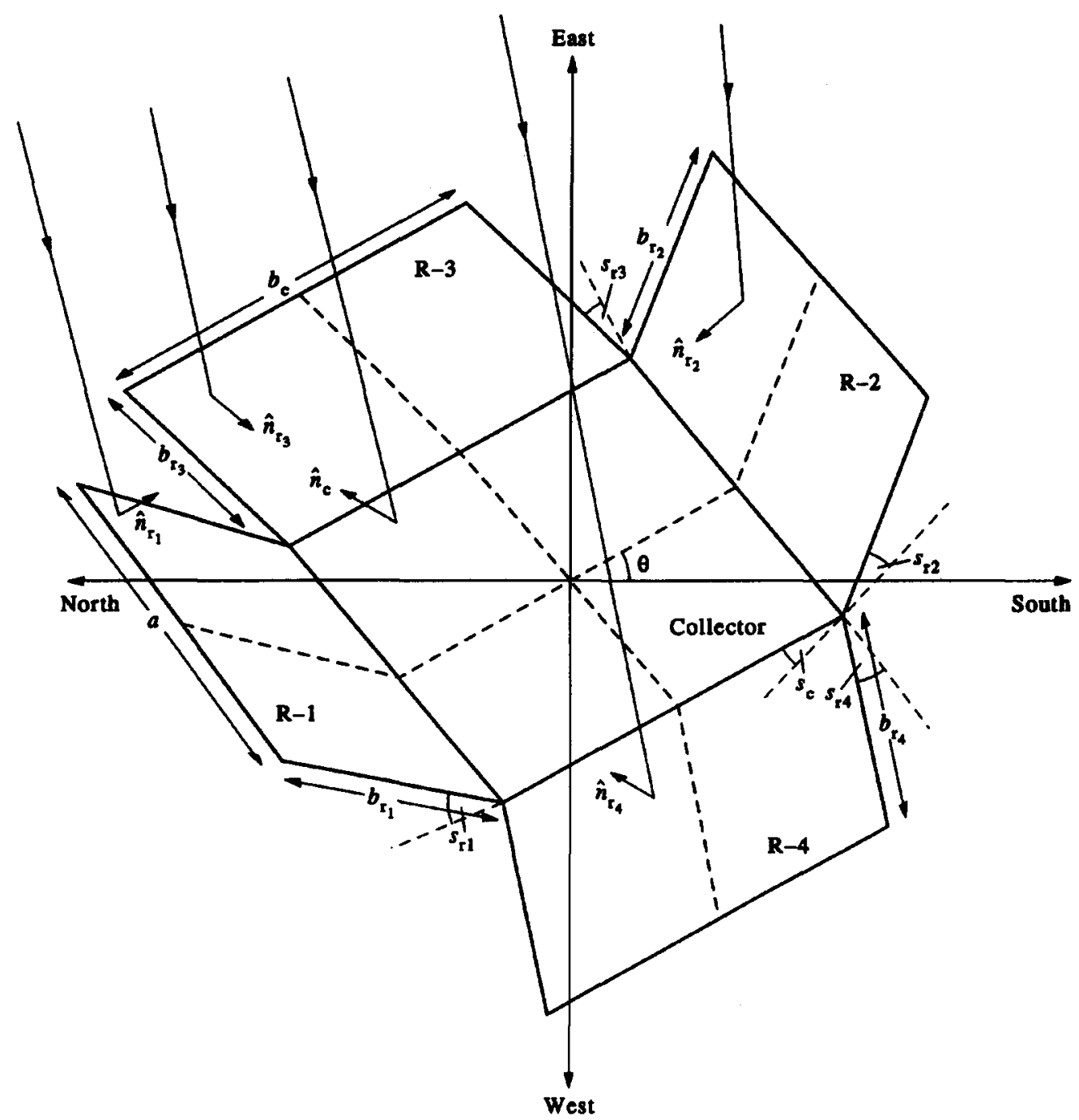

Fig. 1. A schematic representation of the collector-reflector assembly.

Table 1. Values of $A, B$, and $C$ for the representative day of each month.

\begin{tabular}{lccc}
\hline Date & $A\left(\mathrm{~W} / \mathrm{m}^{2}\right)$ & $B$ & $C$ \\
\hline 17 Jan. & 1230 & 0.1420 & 0.0580 \\
16 Feb. & 1217 & 0.1434 & 0.0590 \\
$16 \mathrm{Mar}$. & 1191 & 0.1520 & 0.0690 \\
$15 \mathrm{Apr}$. & 1146 & 0.1750 & 0.0920 \\
15 May & 1110 & 0.1930 & 0.1160 \\
11 June & 1093 & 0.2020 & 0.1300 \\
17 July & 1086 & 0.2066 & 0.1356 \\
16 Aug. & 1102 & 0.2020 & 0.1200 \\
15 Sep. & 1143 & 0.1816 & 0.0980 \\
15 Oct. & 1184 & 0.1630 & 0.0760 \\
14 Nov. & 1214 & 0.1510 & 0.0650 \\
10 Dec. & 1229 & 0.1440 & 0.0590 \\
\hline
\end{tabular}

$$
\begin{aligned}
Q & =\left(1-S_{\mathrm{rc}}\right)\left\{(\alpha \tau)_{\mathrm{bc}} I_{\mathrm{bc}}+(\alpha \tau)_{\mathrm{dc}} I_{\mathrm{dc}}+\rho_{\mathrm{r}}\left[(\alpha \tau)_{\mathrm{rc} 1} I_{\mathrm{br}} f_{\mathrm{rc} 1} \cos \theta_{\mathrm{rc} 1}+(\alpha \tau)_{\mathrm{rc} 2} I_{\mathrm{br} 2} f_{\mathrm{rc} 2} \cos \theta_{\mathrm{rc} 2}\right.\right. \\
& \left.\left.+(\alpha \tau)_{\mathrm{rc} 3} I_{\mathrm{br} 3} f_{\mathrm{rc} 3} \cos \theta_{\mathrm{rc} 3}+(\alpha \tau)_{\mathrm{rc} 4} I_{\mathrm{br} 4} f_{\mathrm{rc} 4} \cos \theta_{\mathrm{rc} 4}\right]\right\}, \\
S_{\mathrm{rc}} & =S_{\mathrm{rc} 1}+S_{\mathrm{rc} 2}+S_{\mathrm{rc} 3}+S_{\mathrm{rc} 4},
\end{aligned}
$$


where the first term in the curly bracket of Eq. (1) stands for direct radiation incident on the collector, the second term is the diffuse component, and the third term is energy received by the collector from the reflectors. The first term within the square bracket is the energy from reflector R-1 absorbed by the collector. Similarly, the 2nd, 3rd, and 4th terms give the reflected energy on the collector due to reflectors R-2, R-3, and R-4, respectively. $S_{\mathrm{rc} 1}, S_{\mathrm{rc} 2}, S_{\mathrm{rc} 3}$, and $S_{\mathrm{rc} 4}$ are shading factors on the collector due to reflectors $R-1, R-2, R-3$, and $R-4$, respectively.

The following relations ${ }^{10}$ are used to evaluate the beam and diffuse components of radiation on the collector and the beam radiation on the reflectors:

$$
\begin{aligned}
I_{\mathrm{bc}} & =I_{\mathrm{DN}} \cos \theta_{\mathrm{c}}, I_{\mathrm{dc}}=C I_{\mathrm{DN}} f_{\mathrm{cs}}, I_{\mathrm{br}}=I_{\mathrm{DN}} \cos \theta_{\mathrm{r}}, \\
I_{\mathrm{DN}} & =A \exp (-B / \sin \beta) ;
\end{aligned}
$$

$A, B$ and $C$ are constants (see Table 1). The shape factor between the collector and the sky is

$$
f_{\mathrm{cs}}=\left(1+\cos s_{\mathrm{c}}\right) / 2 \text {. }
$$

If $\phi$ is the off-south angle of the system, the unit vectors normal to the collector surface and reflectors are, respectively,

$$
\begin{aligned}
& \bar{n}_{\mathrm{c}}=\sin s_{\mathrm{c}} \cos \phi \bar{i}+\sin s_{\mathrm{c}} \sin \phi \bar{j}+\cos s_{\mathrm{c}} \bar{k}, \bar{n}_{\mathrm{r} 1}=\sin s_{\mathrm{r} 1} \cos \phi \bar{i}+\sin s_{\mathrm{r} 1} \sin \phi \bar{j}+\cos s_{\mathrm{r} 1} \bar{k}, \\
& \bar{n}_{\mathrm{r} 2}=-\sin s_{\mathrm{r} 2} \cos \phi \bar{i}-\sin s_{\mathrm{r} 2} \sin \phi \bar{j}+\cos s_{\mathrm{r} 2} \bar{k}, \bar{n}_{\mathrm{r} 3}=\sin s_{\mathrm{r} 3} \sin \phi \bar{i}-\sin s_{\mathrm{r} 3} \cos \phi \bar{j}+\cos s_{\mathrm{r} 3} \bar{k}, \\
& \bar{n}_{\mathrm{r} 4}=-\sin s_{\mathrm{r} 4} \sin \phi \bar{i}+\sin s_{\mathrm{r} 4} \cos \phi \bar{j}+\cos s_{\mathrm{r} 4} \bar{k} .
\end{aligned}
$$

The unit vector in the direction of the incoming beam radiation is

$$
\bar{n}_{\mathrm{b}}=-\cos \beta \cos \gamma \bar{i}-\cos \beta \cos \gamma \bar{j}-\sin \beta \bar{k},
$$

where

$$
\sin \beta=\cos L \cos \delta \cos H+\sin \delta \sin L, \sin \gamma=\cos \delta \sin H / \cos \beta
$$

$\delta$ is the declination angle and is given by $23.45 \sin [360(284+N) / 365]$.

The angles of incidence on the collector and reflectors are

$$
\begin{aligned}
& \cos \theta_{\mathrm{c}}=\sin s_{\mathrm{c}} \cos \beta \cos (\gamma-\phi)+\cos s_{\mathrm{c}} \sin \beta, \cos \theta_{\mathrm{r} 1}=\sin s_{\mathrm{r} 1} \cos \beta \cos (\gamma-\phi)+\cos s_{\mathrm{r} 1} \sin \beta, \\
& \cos \theta_{\mathrm{r} 2}=-\sin s_{\mathrm{r} 2} \cos \beta \cos (\gamma-\phi)+\cos s_{\mathrm{r} 2} \sin \beta, \cos \theta_{\mathrm{r} 3}=-\sin s_{\mathrm{r} 3} \cos \beta \sin (\gamma-\phi)+\cos s_{\mathrm{r} 3} \sin \beta \\
& \cos \theta_{\mathrm{r} 4}=\sin s_{\mathrm{r} 4} \cos \beta \sin (\gamma-\phi)+\cos s_{\mathrm{r} 4} \sin \beta .
\end{aligned}
$$

- The unit vectors in the directions of the reflected rays from the reflectors are, respectively, $\bar{n}_{\mathrm{rc} 1}, \bar{n}_{\mathrm{rc} 2}, \bar{n}_{\mathrm{rc} 3}$, and $\bar{n}_{\mathrm{rc} 4}$ and may be expressed as

$$
\bar{n}_{\mathrm{rc} 1}=u_{1} \bar{i}+v_{1} \bar{j}+w_{1} \bar{k}, \bar{n}_{\mathrm{rc} 2}=u_{2} \bar{i}+v_{2} \bar{j}+w_{2} \bar{k}, \bar{n}_{\mathrm{rc} 3}=u_{3} \bar{i}+v_{3} \bar{j}+w_{3} \bar{k}, \bar{n}_{\mathrm{rc} 4}=u_{4} \bar{i}+v_{4} \bar{j}+w_{4} \bar{k},
$$

where

$$
\begin{aligned}
& u_{1}=-\cos \beta \cos \gamma+2 \cos \theta_{\mathrm{r} 1} \sin s_{\mathrm{r} 1} \cos \phi, v_{1}=-\cos \beta \sin \gamma+2 \cos \theta_{\mathrm{r} 1} \sin s_{\mathrm{r} 1} \sin \phi, \\
& w_{1}=-\sin \beta+2 \cos \theta_{\mathrm{r} 1} \cos s_{\mathrm{r} 1}, \\
& u_{2}=-\cos \beta \cos \gamma-2 \cos \theta_{\mathrm{r} 2} \sin s_{\mathrm{r} 2} \cos \phi, v_{2}=-\cos \beta \sin \gamma-2 \cos \theta_{\mathrm{r} 2} \sin s_{\mathrm{r} 2} \sin \phi, \\
& w_{2}=-\sin \beta+2 \cos \theta_{\mathrm{r} 2} \cos s_{\mathrm{r} 2}
\end{aligned}
$$




$$
\begin{aligned}
& u_{3}=-\cos \beta \cos \gamma+2 \cos \theta_{\mathrm{r} 3} \sin s_{\mathrm{r} 3} \sin \phi, v_{3}=-\cos \beta \sin \gamma-2 \cos \theta_{\mathrm{r} 3} \sin s_{\mathrm{r} 3} \cos \phi, \\
& w_{3}=-\sin \beta+2 \cos \theta_{\mathrm{r} 3} \cos s_{\mathrm{r} 3}, \\
& u_{4}=-\cos \beta \cos \gamma-2 \cos \theta_{\mathrm{r} 4} \sin s_{\mathrm{r} 4} \sin \phi, v_{4}=-\cos \beta \sin \gamma+2 \cos \theta_{\mathrm{r} 4} \sin s_{\mathrm{r} 3} \cos \phi, \\
& w_{4}=-\sin \beta+2 \cos \theta_{\mathrm{r} 4} \cos s_{\mathrm{r} 4} .
\end{aligned}
$$

The expressions for the angles of incidence on the collector for the reflected rays are, respectively,

$$
\begin{aligned}
& \cos \theta_{\mathrm{re} 1}=-u_{1} \sin s_{\mathrm{c}} \cos \phi-v_{1} \sin s_{\mathrm{c}} \sin \phi-w_{1} \cos s_{\mathrm{c}} \\
& \cos \theta_{\mathrm{rc} 2}=-u_{2} \sin s_{\mathrm{c}} \cos \phi-v_{2} \sin s_{\mathrm{c}} \sin \phi-w_{2} \cos s_{\mathrm{c}} \\
& \cos \theta_{\mathrm{re} 3}=-u_{3} \sin s_{\mathrm{c}} \cos \phi-v_{3} \sin s_{\mathrm{c}} \sin \phi-w_{3} \cos s_{\mathrm{c}} \\
& \cos \theta_{\mathrm{re} 4}=-u_{4} \sin s_{\mathrm{c}} \cos \phi-v_{4} \sin s_{\mathrm{c}} \sin \phi-w_{4} \cos s_{\mathrm{c}}
\end{aligned}
$$

\section{EVALUATION OF THE EXCHANGE AND SHADING FACTORS}

The fraction of the total collector area illuminated by reflected radiation from a reflector is the exchange factor $\left(f_{\mathrm{rc}}\right)$. It is a function of collector and reflector tilts, the relative dimensions of the collector-reflector assembly, and the position of the sun in the sky. As shown in Fig. 2, if $\bar{e}_{1}, \bar{e}_{2}, \bar{e}_{3}$, and $\bar{e}_{4}$ are the position vectors of points on the incident solar rays after reflection, then

$$
\bar{e}_{1}=e_{\mathrm{x} 1} \bar{i}+e_{\mathrm{y} 1} \bar{j}+e_{\mathrm{z} 1} \bar{k}, \bar{e}_{2}=e_{\mathrm{x} 2} \bar{i}+e_{\mathrm{y} 2} \bar{j}+e_{\mathrm{z} 2} \bar{k}, \bar{e}_{3}=e_{\mathrm{x} 3} \bar{i}+e_{\mathrm{y} 3} \bar{j}+e_{\mathrm{z} 3} \bar{k}, \bar{e}_{4}=e_{\mathrm{x} 4} \bar{i}+e_{\mathrm{y} 4} \bar{j}+e_{\mathrm{z} 4} \bar{k}
$$

where

$$
\begin{aligned}
& e_{\mathrm{x} 1}=-(a / 2) \sin \phi-b_{\mathrm{r} 1} \cos s_{\mathrm{r} 1} \cos \phi+C_{\mathrm{e} 1} u_{1}, e_{\mathrm{y} 1}=-(a / 2) \cos \phi-b_{\mathrm{r} 1} \cos s_{\mathrm{r} 1} \sin \phi+C_{\mathrm{e} 1} v_{1}, \\
& e_{\mathrm{z} 1}=b_{\mathrm{r} 1} \sin s_{\mathrm{r} 1}+C_{\mathrm{e} 1} w_{1}, \\
& e_{\mathrm{x} 2}=-(a / 2) \sin \phi-b_{\mathrm{r} 2} \cos s_{\mathrm{r} 2} \cos \phi+C_{\mathrm{e} 2} u_{2}, e_{\mathrm{y} 2}=-(a / 2) \cos \phi-b_{\mathrm{r} 2} \cos s_{\mathrm{r} 2} \sin \phi+C_{\mathrm{e} 2} v_{2}, \\
& e_{\mathrm{z} 2}=b_{\mathrm{r} 2} \sin s_{\mathrm{r} 2}+C_{\mathrm{e} 2} w_{2}, \\
& e_{\mathrm{x} 3}=-\left(b_{\mathrm{c}} / 2\right) \cos \phi-b_{\mathrm{r} 3} \cos s_{\mathrm{r} 3} \sin \phi+C_{\mathrm{e} 3} u_{3}, e_{\mathrm{y} 3}=-\left(b_{\mathrm{c}} / 2\right) \sin \phi-b_{\mathrm{r} 3} \cos s_{\mathrm{r} 3} \cos \phi+C_{\mathrm{e} 3} v_{3}, \\
& e_{\mathrm{z} 3}=b_{\mathrm{r} 3} \sin s_{\mathrm{r} 3}+C_{\mathrm{e} 3} w_{3}, \\
& e_{\mathrm{x} 4}=-\left(b_{\mathrm{c}} / 2\right) \cos \phi-b_{\mathrm{r} 4} \cos s_{\mathrm{r} 4} \sin \phi+C_{\mathrm{e} 4} u_{4}, e_{\mathrm{y} 4}=-\left(b_{\mathrm{c}} / 2\right) \sin \phi-b_{\mathrm{r} 4} \cos s_{\mathrm{r} 4} \cos \phi+C_{\mathrm{e} 4} v_{4}, \\
& e_{\mathrm{z} 4}=b_{\mathrm{r} 4} \sin s_{\mathrm{r} 4}+C_{\mathrm{e} 4} w_{4} .
\end{aligned}
$$

Here,

$$
\begin{aligned}
& C_{\mathrm{e} 1}=b_{\mathrm{r} 1} \sin \left(s_{\mathrm{r} 1}-s_{\mathrm{c}}\right) / \cos \theta_{\mathrm{rc} 1}, \\
& C_{\mathrm{e} 2}=b_{\mathrm{r} 2} \sin \left(s_{\mathrm{r} 2}+s_{\mathrm{c}}\right) / \cos \theta_{\mathrm{rc} 2}, \\
& C_{\mathrm{e} 3}=b_{\mathrm{r} 3} \sin s_{\mathrm{r} 3} / \cos \theta_{\mathrm{re} 3}, \\
& C_{\mathrm{e} 4}=b_{\mathrm{r} 4} \sin s_{\mathrm{r} 4} / \cos \theta_{\mathrm{rc} 4} .
\end{aligned}
$$

Expressions for the exchange factors with respect to different regions of the reflectors are given in Tables 2-5. Shading of reflectors for radiation to the collector is calculated similarly. Here, instead of taking reflected rays from points $E_{1}$ to $E_{4}$, we consider the incoming beam of solar radiation as passing from these points and falling on the collector. The position vectors of points for which the incoming beams are incident on the collector are 


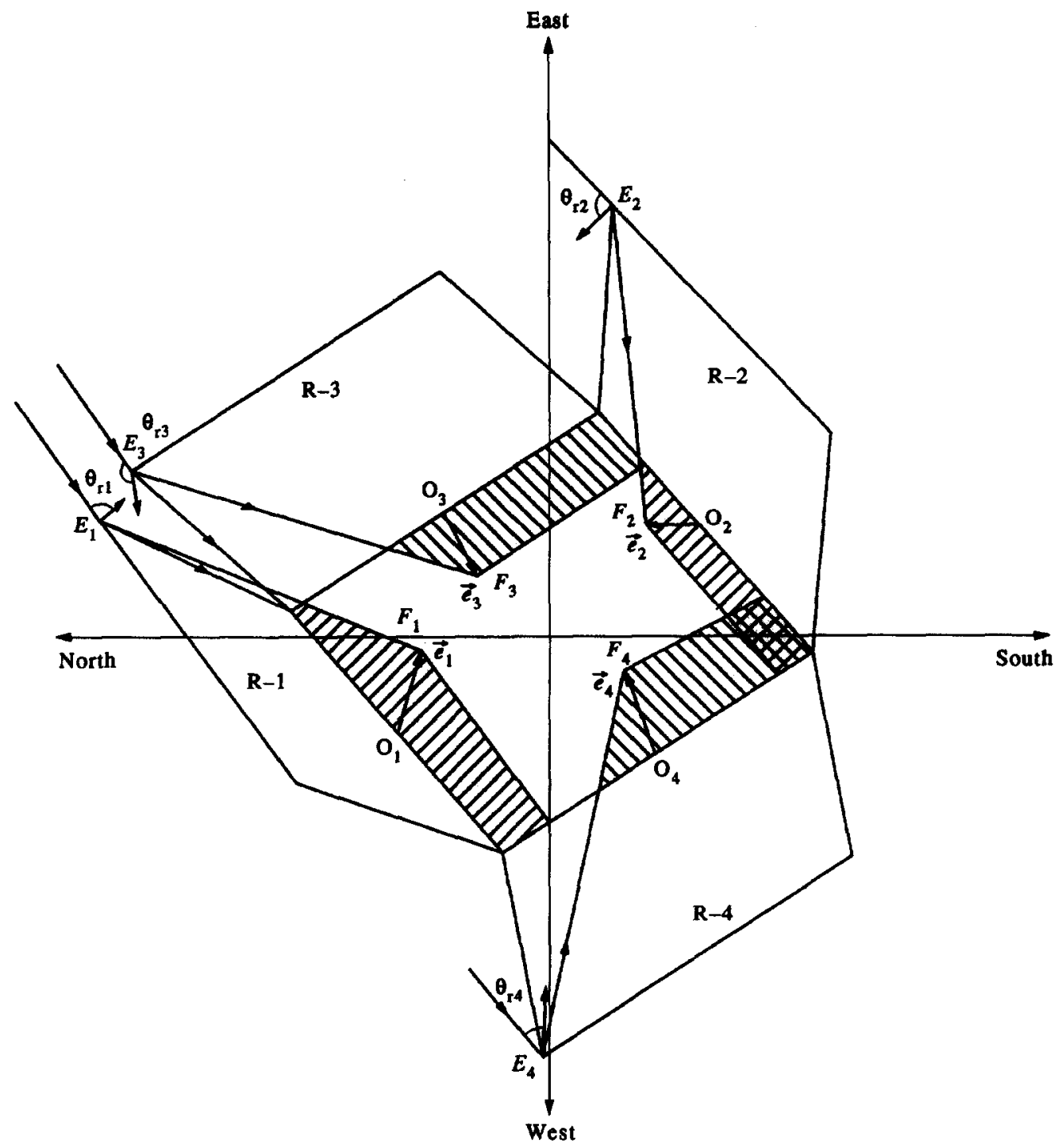

Fig. 2. Schematic representation of the reflected rays from the reflectors and falling on the collector.

$$
\begin{aligned}
& \bar{e}_{1}^{\prime}=e_{\mathrm{x} 1}^{\prime} \bar{i}+e_{\mathrm{y} 1}^{\prime} \bar{j}+e_{\mathrm{z} 1}^{\prime} \bar{k}, \bar{e}_{2}^{\prime}=e_{x 2}^{\prime} \bar{i}+e_{\mathrm{y} 2}^{\prime} \bar{j}+e_{\mathrm{z} 2}^{\prime} \bar{k}, \bar{e}_{3}^{\prime}=e_{\mathrm{x} 3}^{\prime} \bar{i}+e_{\mathrm{y} 3}^{\prime} \bar{j}+e_{\mathrm{z} 3}^{\prime} \bar{k}, \\
& \bar{e}_{4}^{\prime}=e_{\mathrm{x} 4}^{\prime} \bar{i}+e_{\mathrm{y} 4}^{\prime} \bar{j}+e_{\mathrm{z} 4}^{\prime} \bar{k},
\end{aligned}
$$

where

$e_{\mathrm{x} 1}^{\prime}=-(a / 2) \sin \phi-b_{\mathrm{r} 1} \cos s_{\mathrm{r} 1} \cos \phi-C_{\mathrm{e} 1}^{\prime} \cos \beta \cos \gamma, e_{\mathrm{y} 1}^{\prime}=-(a / 2) \cos \phi-b_{\mathrm{r} 1} \cos s_{\mathrm{r} 1} \sin \phi-C_{\mathrm{e} 1}^{\prime} \cos \beta \sin \gamma$,

$e_{\mathrm{z} 1}^{\prime}=b_{\mathrm{r} 1} \sin s_{\mathrm{r} 1}-C_{\mathrm{e} 1}^{\prime} \sin \beta$,

$e_{\mathrm{x} 2}^{\prime}=-(a / 2) \sin \phi-b_{\mathrm{r} 2} \cos s_{\mathrm{r} 2} \cos \phi-C_{\mathrm{e} 2}^{\prime} \cos \beta \cos \gamma, e_{\mathrm{y} 2}^{\prime}=-(a / 2) \cos \phi-b_{\mathrm{r} 2} \cos s_{\mathrm{r} 2} \sin \phi-C_{\mathrm{e} 2}^{\prime} \cos \beta \sin \gamma$,

$e_{\mathrm{z} 2}^{\prime}=b_{\mathrm{r} 2} \sin s_{\mathrm{r} 2}-C_{\mathrm{e} 2}^{\prime} \sin \beta$,

$$
\begin{aligned}
& e_{\mathrm{x} 3}^{\prime}=-\left(b_{\mathrm{c}} / 2\right) \cos \phi-b_{\mathrm{r} 3} \cos s_{\mathrm{r} 3} \sin \phi-C_{\mathrm{e} 3}^{\prime} \cos \beta \cos \gamma, \\
& e_{\mathrm{y} 3}^{\prime}=-\left(b_{\mathrm{c}} / 2\right) \sin \phi-b_{\mathrm{r} 3} \cos s_{\mathrm{r} 3} \cos \phi-C_{\mathrm{e} 3}^{\prime} \cos \beta \sin \gamma, \\
& e_{\mathrm{z} 3}^{\prime}=b_{\mathrm{r} 3} \sin s_{\mathrm{r} 3}-C_{\mathrm{e} 3}^{\prime} \sin \beta, \\
& e_{\mathrm{x} 4}^{\prime}=-\left(b_{\mathrm{c}} / 2\right) \cos \phi-b_{\mathrm{r} 4} \cos s_{\mathrm{r} 4} \sin \phi-C_{\mathrm{e} 4}^{\prime} \cos \beta \cos \gamma,
\end{aligned}
$$


Table 2. Values of the exchange factors with specified boundary conditions between the reflector R-1 and the collector for six different regions (defined by the boundary conditions).

\begin{tabular}{|c|c|c|}
\hline Region & Boundary conditions & Exchange factor $\left(f_{\mathrm{rcl}}\right)$ \\
\hline 1 & $\begin{aligned} R_{1} \geqslant 0, S_{1} & <-0.5 \\
\left(\mathrm{R}_{1}+\mathrm{S}_{1} \mathrm{~W}_{1}\right) & <0.5 \mathrm{~W}_{1}\end{aligned}$ & $0.5 R_{1} / W_{1}\left(0.5-S_{1}\right)$ \\
\hline 2 & $\begin{array}{c}R_{1}>W_{1}, S_{1} \leqslant 0.5 \\
\left(R_{1}+S_{1} W_{1}\right) \geqslant 0.5 W_{1}\end{array}$ & $0.5\left[2-\left(0.5-S_{1}\right) W_{1} / R_{1}\right]$ \\
\hline 3 & $\begin{array}{c}-0.5 \leqslant S_{1} \leqslant 0.5 \\
0 \leqslant R_{1} \leqslant W_{1}\end{array}$ & $0.5\left(1.5+S_{1}\right) R_{1} / W_{1}$ \\
\hline 4 & $\begin{array}{c}S_{1}>0.5, R_{1} \geqslant W_{1} \\
\left(R_{1}-S_{1} W_{1}\right)>-0.5 W_{1}\end{array}$ & $0.5\left[2-\left(S_{1}-0.5\right) W_{1} / R_{1}\right]$ \\
\hline 5 & $\begin{array}{c}0.5<S_{1} \leqslant 1.5 \\
0 \leqslant \mathrm{R}_{1}<\mathrm{W}_{1}\end{array}$ & $0.5 R_{1}\left(2.5-S_{1}\right) / W_{1}$ \\
\hline 6 & $\begin{array}{c}S_{1}>0.5, R_{1} \geqslant 0 \\
\left(R_{1}-S_{1} W_{1}\right)<-0.5 W_{1}\end{array}$ & $0.5 R_{1} / W_{1}\left(S_{1}-0.5\right)$ \\
\hline
\end{tabular}

$S_{1}=-\left(\mathrm{e}_{\mathrm{x} 1} / \mathrm{a}\right) \sin \phi+\left(e_{\mathrm{y} 1} / a\right) \cos \phi, R_{1}=\left(e_{\mathrm{x} 1} / a\right) \cos \phi+\left(e_{\mathrm{y} 1} / a\right) \sin \phi, S_{1}^{\prime}=-\left(e_{\mathrm{x} 1}^{\prime} / a\right) \sin \phi+\left(e_{\mathrm{y} 1}^{\prime} / a\right) \cos \phi, R_{1}^{\prime}=\left(e_{\mathrm{x} 1}^{\prime} / a\right) \cos \phi+$ $\left(e_{\mathrm{y} 1}^{\prime} / a\right) \sin \phi, W_{1}=\left(b_{\mathrm{c}} / a\right) \cos s_{\mathrm{c}}$.

Table 3. Values of the exchange factors with specified boundary conditions between the reflector R-2 and the collector for six different regions (defined by the boundary conditions).

\begin{tabular}{|c|c|c|}
\hline Region & Boundary conditions & Exchange factor $\left(f_{\mathrm{rc} 2}\right)$ \\
\hline 1 & $\begin{array}{c}R_{2} \leqslant 0, S_{2}<-0.5 \\
\left(R_{2}+S_{2} W_{2}\right)>0.5 W_{2}\end{array}$ & $0.5 R_{2} / W_{2}\left(0.5-S_{2}\right)$ \\
\hline 2 & $\begin{array}{c}R_{2}<W_{2}, S_{2} \leqslant 0.5 \\
\left(R_{2}+S_{2} W_{2}\right)<0.5 W_{2}\end{array}$ & $0.5\left[2-\left(0.5-S_{2}\right) W_{2} / R_{2}\right]$ \\
\hline 3 & $\begin{array}{c}-0.5 \leqslant S_{2} \leqslant 0.5 \\
0 \geqslant R_{2}>W_{2}\end{array}$ & $0.5\left(1.5+S_{2}\right) R_{2} / W_{2}$ \\
\hline 4 & $\begin{array}{c}S_{2}>0.5, R_{2} \leqslant W_{2} \\
\left(R_{2}-S_{2} W_{2}\right)<-0.5 W_{2}\end{array}$ & $0.5\left[2-\left(S_{2}-0.5\right) W_{2} / R_{2}\right]$ \\
\hline 5 & $\begin{array}{c}0.5<S_{2}<1.5 \\
0 \geqslant R_{2}>W_{2}\end{array}$ & $0.5 R_{2}\left(2.5-S_{2}\right) / W_{2}$ \\
\hline 6 & $\begin{array}{c}S_{2}>0.5, R_{2} \leqslant 0 \\
\left(R_{2}-S_{2} W_{2}\right)>-0.5 W_{2}\end{array}$ & $0.5 R_{2} / W_{2}\left(S_{2}-0.5\right)$ \\
\hline
\end{tabular}

$S_{2}=-\left(e_{x 2} / a\right) \sin \phi+\left(e_{y 2} / a\right) \cos \phi, R_{1}=\left(e_{x 2} / a\right) \cos \phi-\left(e_{y 2} / a\right) \sin \phi, S_{2}^{\prime}=\left(e_{x 2}^{\prime} / a\right) \sin \phi+\left(e_{y 2}^{\prime} / a\right) \cos \phi, R_{2}^{\prime}=\left(e_{x 2}^{\prime} / a\right) \cos \phi-$ $\left(e_{\mathrm{y} 2}^{\prime} / a\right) \sin \phi, W_{2}=\left(b_{\mathrm{c}} / a\right) \cos s_{\mathrm{c}}$.

$$
\begin{aligned}
& e_{\mathrm{y} 4}^{\prime}=-\left(b_{\mathrm{c}} / 2\right) \sin \phi-b_{\mathrm{r} 4} \cos s_{\mathrm{r} 4} \cos \phi-C_{\mathrm{e} 4}^{\prime} \cos \beta \sin \gamma, \\
& e_{\mathrm{z} 4}^{\prime}=b_{\mathrm{r} 4} \sin s_{\mathrm{r} 4}-C_{\mathrm{e} 4}^{\prime} \sin \beta .
\end{aligned}
$$

Here,

$$
\begin{aligned}
& C_{\mathrm{e} 1}^{\prime}=b_{\mathrm{r} 1} \sin \left(s_{\mathrm{r} 1}-s_{\mathrm{c}}\right) / \cos \theta_{\mathrm{c}}, \\
& C_{\mathrm{e} 2}^{\prime}=b_{\mathrm{r} 2} \sin \left(s_{\mathrm{r} 2}+s_{\mathrm{c}}\right) / \cos \theta_{\mathrm{c}}, \\
& C_{\mathrm{e} 3}^{\prime}=b_{\mathrm{r} 3} \sin s_{\mathrm{r} 3} / \cos \theta_{\mathrm{c}}, \\
& C_{\mathrm{e} 4}^{\prime}=b_{\mathrm{r} 4} \sin s_{\mathrm{r} 4} / \cos \theta_{\mathrm{c}} .
\end{aligned}
$$


Table 4. Values of the exchange factors with specified boundary conditions between the reflector R-3 and the collector for six different regions (defined by the boundary conditions).

\begin{tabular}{ccc}
\hline Region & Boundary conditions & Exchange factor $\left(f_{\mathrm{rc}}\right)$ \\
\hline 1 & $R_{3} \leqslant 0, S_{3}<-0.5$ & $0.5 R_{3} / W_{3}\left(0.5-S_{3}\right)$ \\
& $\left(R_{3}+S_{3} W_{3}\right)>0.5 W_{3}$ & \\
2 & $R_{3} \leqslant W_{3}, S_{3} \leqslant 0.5$ & $0.5\left[2-\left(0.5-S_{3}\right) W_{3} / R_{3}\right]$ \\
& $\left(R_{3}+S_{3} W_{3}\right) \leqslant 0.5 W_{3}$ & \\
3 & $-0.5 \leqslant S_{3}<0.5$ & $0.5\left(1.5+S_{3}\right) R_{3} / W_{3}$ \\
& $0 \geqslant 6 R_{3}>W_{3}$ & \\
4 & $S_{3}>0.5, R_{3} \leqslant W_{3}$ & $0.5\left[2-\left(S_{3}-0.5\right) W_{3} / R_{3}\right]$ \\
5 & $\left(R_{3}-S_{3} W_{3}\right)<-0.5 W_{3}$ & \\
6 & $0.5<S_{3} \leqslant 1.5$ & $0.5 R_{3}\left(2.5-S_{3}\right) / W_{3}$ \\
$0>R_{3}>W_{3}$ & \\
& $S_{3}>0.5, R_{3} \leqslant 0$ & $0.5 R_{3} / W_{3}\left(S_{3}-0.5\right)$ \\
\hline
\end{tabular}

$S_{3}=-\left(e_{x 3} / a\right) \cos \phi-\left(e_{y 3} / a\right) \sin \phi, R_{3}=-\left(e_{x 3} / a\right) \sin \phi-\left(e_{y 3} / a\right) \cos \phi, S_{3}^{\prime}=\left(e_{x}^{\prime} / a\right) \cos \phi-\left(e_{y 3}^{\prime} / a\right) \sin \phi, R_{3}^{\prime}=-\left(e_{x}^{\prime} / a\right) \sin \phi-$ $\left(e_{y 3}^{\prime} / a\right) \cos \phi, W_{3}=-1$.

Table 5. Values of the exchange factors with specified boundary conditions between the reflector R-4 and the collector for six different regions (defined by the boundary conditions).

\begin{tabular}{ccc}
\hline Region & Boundary conditions & Exchange factor $\left(f_{\text {rc }}\right)$ \\
\hline 1 & $R_{4} \geqslant 0, S_{4}<-0.5$ & $0.5 R_{4} / W_{4}\left(0.5-S_{4}\right)$ \\
& $\left(R_{4}+S_{4} W_{4}\right)<0.5 W_{4}$ & \\
2 & $R_{4}>W_{4}, S_{4} \leqslant 0.5$ & \\
$\left(R_{4}+S_{4} W_{4}\right)>0.5 W_{4}$ & $0.5\left[2-\left(0.5-S_{4}\right) W_{4} / R_{4}\right]$ \\
3 & $-0.5 \leqslant S_{4} \leqslant 0.5$ & \\
& $0 \leqslant R_{4} \leqslant W 4_{4}$ & $0.5\left(1.5+S_{4}\right) R_{4} / W_{4}$ \\
4 & $S_{4}>0.5, R_{4} \geqslant W_{4}$ \\
5 & $\left(R_{4}-S_{4} W_{4}\right)>-0.5 W_{4}$ & \\
& $0.5<S_{4} \leqslant 1.5$ & $0.5\left[2-\left(S_{4}-0.5\right) W_{4} / R_{4}\right]$ \\
$0 \leqslant R_{4}<W_{4}$ & $0.5 R_{4}\left(2.5-S_{4}\right) / W_{4}$ \\
6 & $S_{4}>0.5, R_{4} \geqslant 0$ & \\
& $\left(R_{4}-S_{4} W_{4}\right)<-0.5 W_{4}$ & $0.5 R_{4} / W_{4}\left(S_{4}-0.5\right)$ \\
\hline
\end{tabular}

$S_{4}=-\left(e_{\mathrm{x} 4} / \mathrm{a}\right) \sin \phi+\left(e_{\mathrm{y}} / a\right) \cos \phi, R_{1}=\left(e_{\mathrm{x} 4} / a\right) \cos \phi+\left(e_{\mathrm{y} 4} / a\right) \sin \phi, S_{4}^{\prime}=-\left(e_{\mathrm{x}}^{\prime} / a\right) \sin \phi+\left(e_{\mathrm{y} 4}^{\prime} / a\right) \cos \phi, R_{4}^{\prime}=\left(e_{\mathrm{x}}^{\prime} / a\right) \cos \phi+$ $\left(e_{y 4}^{\prime} / a\right) \sin \phi, W_{4}=-1$.

The transmittance-absorptance product $(\tau \alpha)$ is

$$
(\tau \alpha)=1.03-0.11 / \cos \theta_{c} \text { for } \cos \theta_{c} \geqslant 0.175,(\tau \alpha)=3.694-2.353 \theta_{c} \text { for } \cos \theta_{c}<0.175 \text {. }
$$

\section{RESULTS AND DISCUSSION}

Numerical calculations have been carried out for May and December in a typical year for Delhi. The collector tilt with respect to the horizontal is zero degree $\left(s_{\mathrm{c}}=0^{\circ}\right)$ and the system faces due south $\left(\phi=0^{\circ}\right)$. Numerical values for other parameters are $a=1.0 \mathrm{~m}, b_{\mathrm{e}}=b_{\mathrm{r} 1}=b_{\mathrm{r} 2}=b_{\mathrm{r} 3}=b_{\mathrm{r} 4}=1.0 \mathrm{~m}$. The values of $s_{\mathrm{r} 1}, s_{\mathrm{r} 2}, s_{\mathrm{r} 3}$ and $s_{\mathrm{r} 4}$ are selected for maximum solar gain. The analysis has three steps.

Step I-The tilts of reflectors R-3 and R-4 ( $s_{\mathrm{r} 3}$ and $\left.s_{\mathrm{r} 4}\right)$ with respect to the horizontal are $0^{\circ}$. The total Etr 20-6-0 


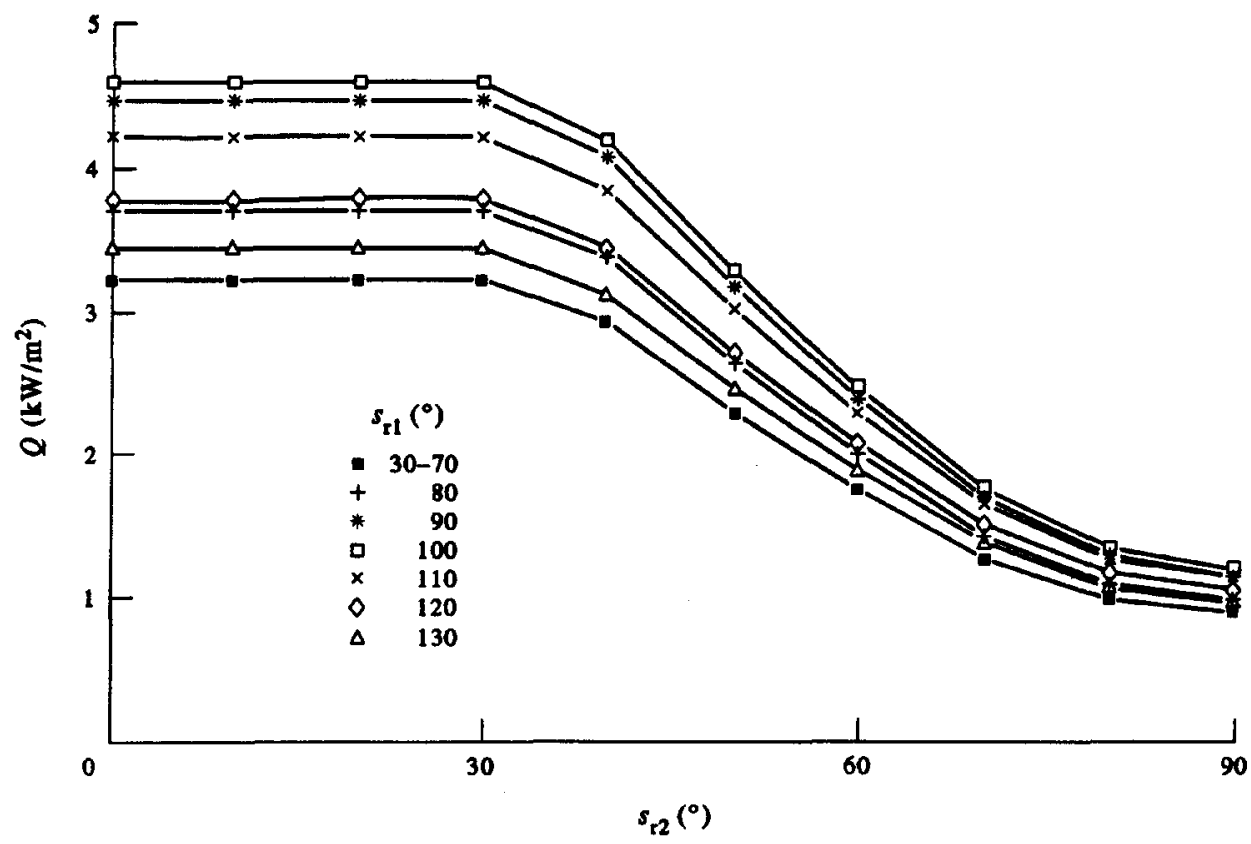

Fig. 3. Variation of the total energy absorbed by the collector with $s_{\mathrm{r} 2}$ for different values of $s_{\mathrm{r} 1}$ for December; $s_{\mathrm{c}}=0^{\circ}, s_{\mathrm{r} 3}=0^{\circ}, s_{\mathrm{r} 4}=0^{\circ}$.

energy $(Q)$ absorbed by the collector during sunshine hours is plotted in Fig. 3 as functions of $s_{\mathrm{r} 1}$ and $s_{\mathrm{r} 2}$ for December. The optimum tilts of reflectors R-1 and R-2 with respect to the horizontal are 100 and $20^{\circ}$, respectively. Figure 4 is used to evaluate optimum tilts for reflectors R-1 and R-2 for May; the optimum values of $s_{\mathrm{r} 1}$ and $s_{\mathrm{r} 2}$ for maximum solar gain on the collector for May are both $60^{\circ}$.

Step II-The tilts of reflectors R-1 and R-2 ( $s_{\mathrm{r} 1}$ and $s_{\mathrm{r} 2}$ ) remain fixed at their optimum values for December and May as found in Step I. The tilts of refiectors R-3 and R-4 ( $s_{\mathrm{r} 3}$ and $\left.s_{\mathrm{r} 4}\right)$ are varied to make the solar gain on the collector a maximum. Figures 5 and 6 show the effects of reflectors R-3

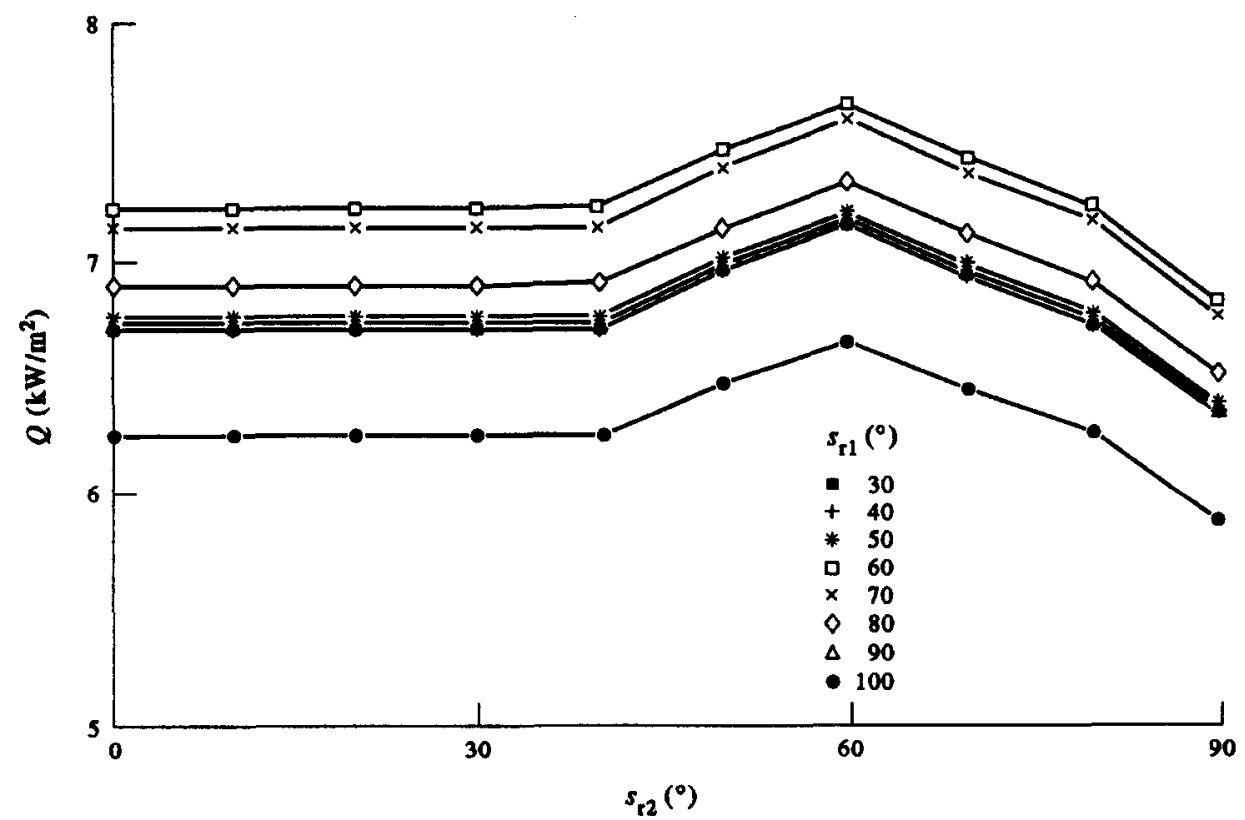

Fig. 4. Variation of the total energy absorbed by the collector with $s_{\mathrm{r} 2}$ for different values of $s_{\mathrm{r} 1}$ for May; $s_{\mathrm{c}}=0^{\circ}, s_{\mathrm{r} 3}=0^{\circ}, s_{\mathrm{r} 4}=0^{\circ}$. 


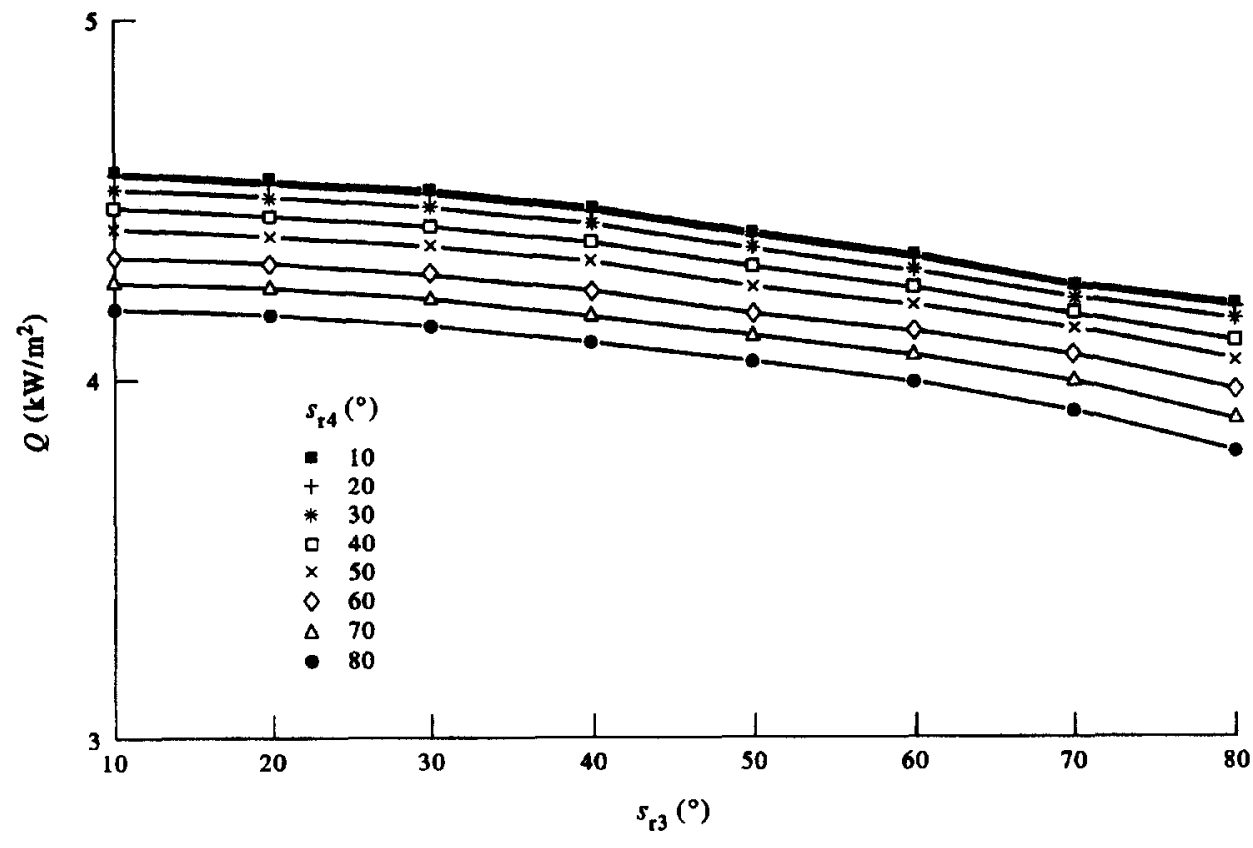

Fig. 5. Variation of the total energy absorbed by the collector with $s_{\mathrm{r} 3}$ for different values of $s_{\mathrm{r} 4}$ for December; $s_{\mathrm{c}}=0^{\circ}, s_{\mathrm{r} 1}=100^{\circ}, s_{\mathrm{r} 2}=20^{\circ}$.

and R-4 on the solar gain of the collector for December and May, respectively. It is evident that the increase in the solar gain of the collector caused by reflectors R-3 and R-4 is negligible. This result is due to the fact that when the solar gain from R-3 increases, that from R-4 decreases because of shadowing and conversely.

Step III-The values of $s_{\mathrm{r} 1}$ and $s_{\mathrm{r} 2}$ are again fixed at their optimum values while $s_{\mathrm{r} 3}$ and $s_{\mathrm{r} 4}$ are varied as in Step II. Shadowing of reflectors R-3 and R-4 is avoided if, before noon, $s_{\mathrm{r} 3}=0^{\circ}$ and, for the afternoon, $s_{\mathrm{r} 4}=0^{\circ}$. The increases in the solar gain for these conditions due to reflectors R-3 and R-4

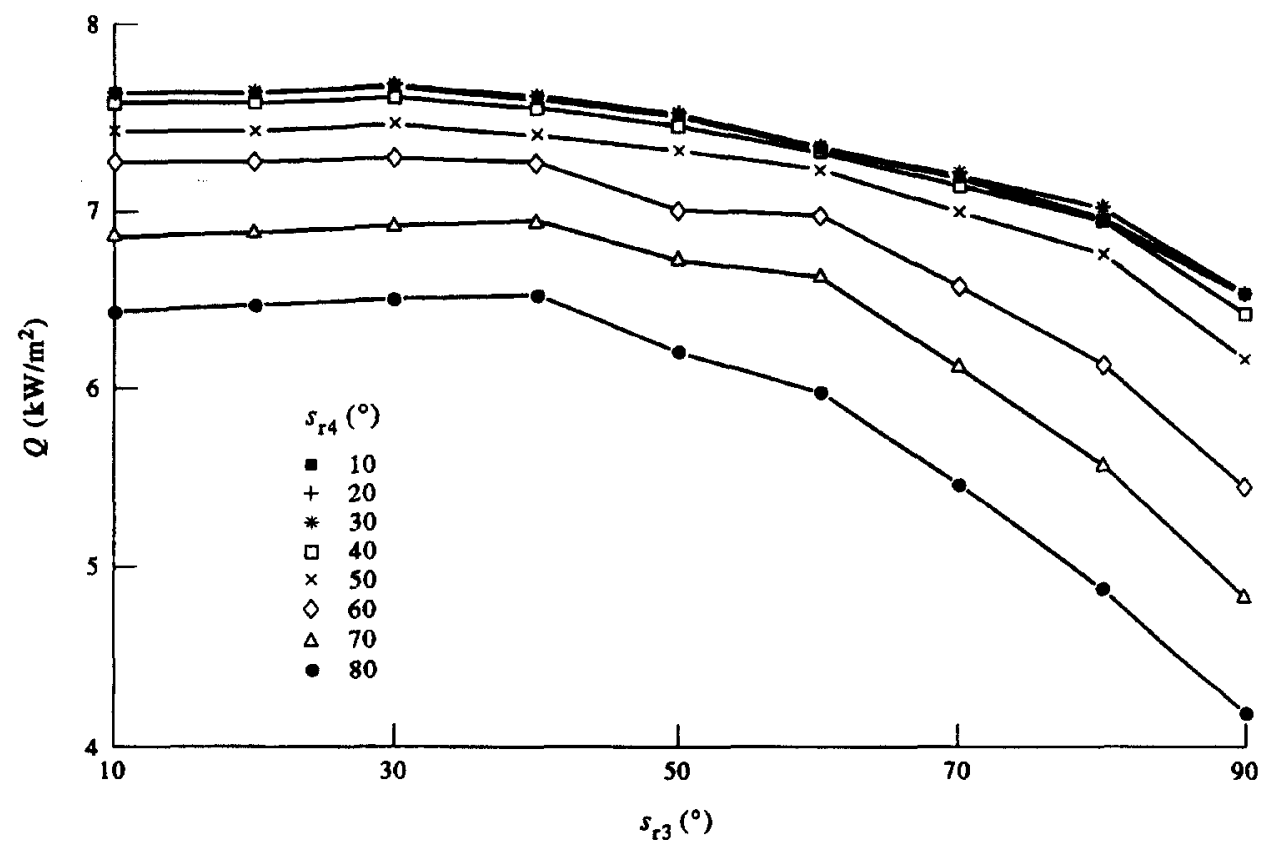

Fig. 6. Variation of the total energy absorbed by the collector with $s_{\mathrm{r} 3}$ for different values of $s_{\mathrm{r} 4}$ for May; $s_{\mathrm{c}}=0^{\circ}, s_{\mathrm{r} 3}=60^{\circ}, s_{\mathrm{r} 4}=60^{\circ}$ 
for December and May are plotted in Figs. 7 and 8. It is seen that reflectors R-3 and R-4 are more effective in May than in December. The increase in the solar gain reaches $36 \%$ in May and $56 \%$ in December. Table 6 shows comparisons of the percentages of solar gains for the three steps taken.

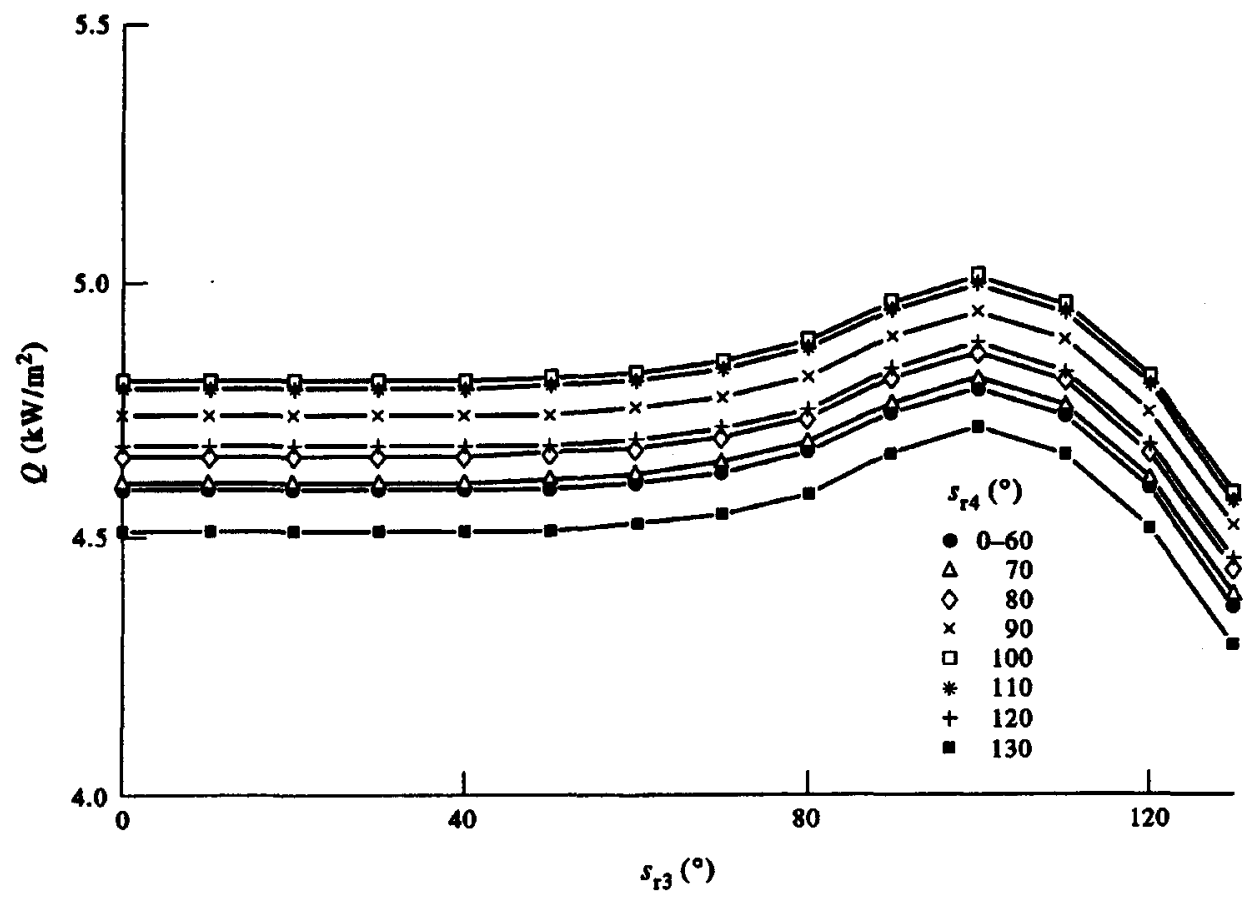

Fig. 7. Variation of the total energy absorbed by the collector with $s_{\mathrm{r} 3}$ for different values of $s_{\mathrm{r} 4}$ for December; $s_{\mathrm{c}}=0^{\circ}, s_{\mathrm{r} 1}=100^{\circ}, s_{\mathrm{r} 2}=20^{\circ}$; before 12 noon, $s_{\mathrm{r} 3}=0^{\circ}$ and after 12 noon, $s_{\mathrm{r} 4}=0^{\circ}$.

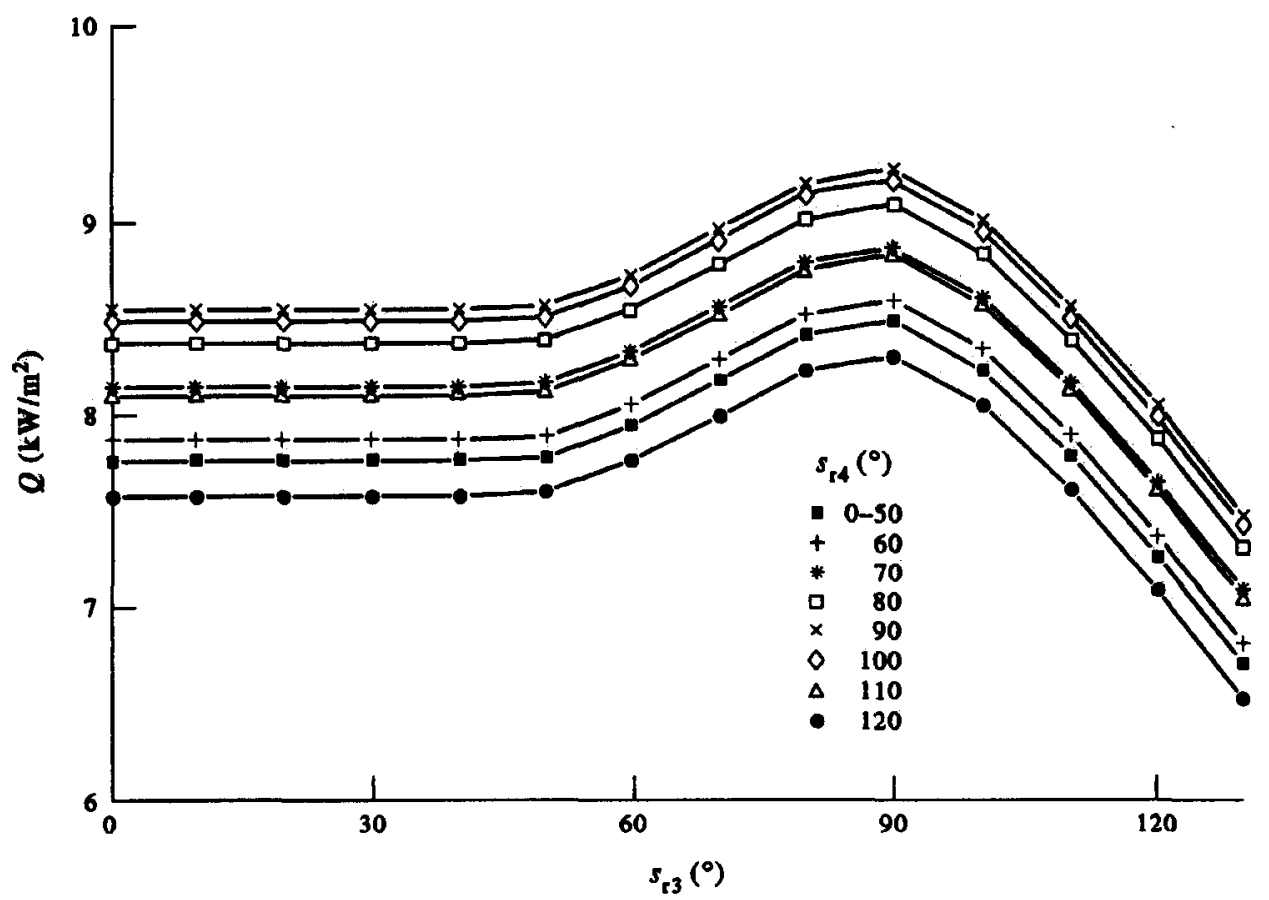

Fig. 8. Variation of the total energy absorbed by the collector with $s_{\mathrm{r} 3}$ for different values of $s_{\mathrm{r} 4}$ for May; $s_{\mathrm{c}}=0^{\circ}, s_{\mathrm{r} 3}=60^{\circ}, s_{\mathrm{r} 4}=60^{\circ}$; before 12 noon, $s_{\mathrm{r} 3}=0^{\circ}$ and after 12 noon, $s_{\mathrm{r} 4}=0^{\circ}$. 
Table 6. Percentage increments of the solar gain on the collector for selected conditions.

\begin{tabular}{|c|c|c|c|c|c|c|c|c|}
\hline Step & Month & $s_{r 1}\left({ }^{\circ}\right)$ & $s_{\mathrm{r} 2}\left({ }^{\circ}\right)$ & $S_{\mathrm{r} 3}\left({ }^{\circ}\right)$ & $S_{\mathrm{r} 4}\left({ }^{\circ}\right)$ & $Q_{0}$ & $Q_{m}$ & $\begin{array}{c}\text { Solar gain } \\
(\%)\end{array}$ \\
\hline \multirow{2}{*}{ I } & Dec & 100 & 20 & 0 & 0 & 3200 & 4591 & 44 \\
\hline & May & 60 & 60 & 0 & 0 & 6836 & 7761 & 14 \\
\hline \multirow{2}{*}{ II } & Dec & 100 & 20 & 0 & 0 & 3200 & 4591 & 44 \\
\hline & May & 60 & 60 & 30 & 30 & 6707 & 7673 & 15 \\
\hline \multirow{2}{*}{ III } & Dec & 100 & 20 & 100 & 100 & 3200 & 5007 & 56 \\
\hline & May & 60 & 60 & 90 & 90 & 6836 & 9247 & 36 \\
\hline
\end{tabular}

Acknowledgement-One of the authors (R. K.) thanks Hrishikesan for fruitful discussions during the present work.

\section{REFERENCES}

1. H. Tabor, Sol. Energy 10, 111 (1966).

2. D. K. McDaniels, D. H. Lowndes, H. M. Mathew, J. Reynolds, and R. Gray, Sol. Energy 17, 277 (1975).

3. S. C. Seitel, Sol. Energy 17, 291 (1975).

4. S. Baker, D. K. McDaniels, H. D. Kahen, and D. H. Lowndes, Sol. Energy 20, 415 (1978).

5. K. D. Mannan and R. D. Banneret, "Optimal Geometries for One- and Two-Faced Symmetric Side Wall Booster," presented at a flat-plate solar conference, Orlando, FL (28 Feb.-2 March 1977).

6. S. L. Grassie and N. R. Sheridean, Sol. Energy 19, 663 (1977).

7. I. S. Taha and S. M. Eldighidy, Sol. Energy 25, 373 (1980).

8. H. P. Garg and D. S. Hrishikesan, Sol. Energy 40(4), 295 (1988).

9. S. C. Kaushik, R. Kumar, S. Chandra, and S. Kaul, "Solar Collector-Refiector System; Analytical Study and Performance Evaluation," Int. J. Sol. Energy (in press).

10. J. A. Duffie and W. A. Beckman, Solar Engineering of Thermal Processes, Wiley, New York, NY (1984).

\section{NOMENCLATURE}

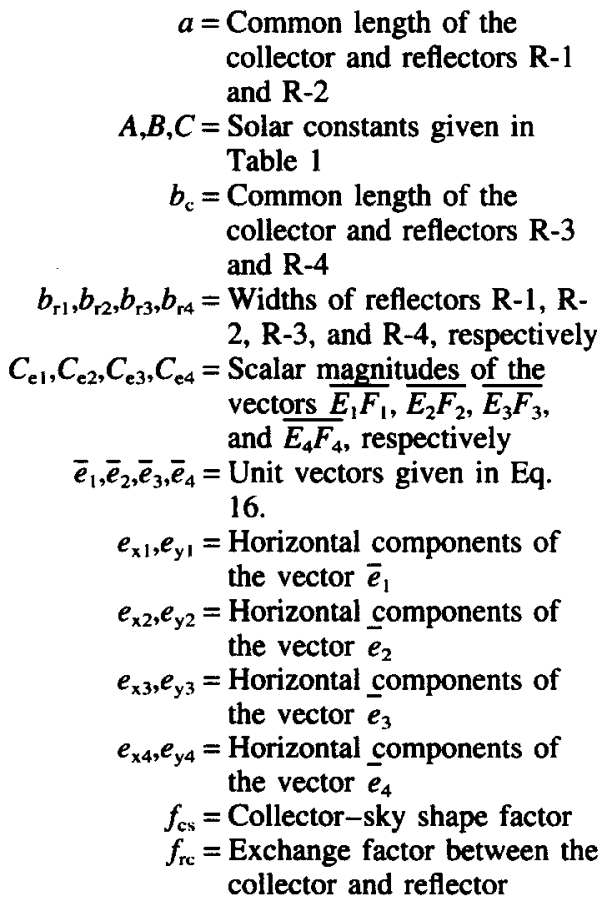

$$
\begin{aligned}
& H=\text { Hour angle } \\
& I=\text { Incident solar intensity } \\
& \left(\mathrm{W} / \mathrm{m}^{2}\right) \\
& \bar{i} \bar{j}, \vec{k}=\text { Unit vectors along the } \mathrm{x}, \mathrm{y} \text {, } \\
& \text { and } z \text { axes, respectively } \\
& L=\text { Latitude of the location } \\
& N=\text { Day of the year } \\
& \bar{n}_{\mathrm{b}}=\text { Unit vector in the direction } \\
& \text { of beam radiation } \\
& \bar{n}_{\mathrm{c}}=\text { Unit vector normal to the } \\
& \text { collector surface } \\
& \bar{n}_{\mathrm{r} 1}, \bar{n}_{\mathrm{r} 2}, \bar{n}_{\mathrm{r} 3}, \bar{n}_{\mathrm{r} 4}=\text { Unit vectors normal to } \\
& \text { reflectors } \mathbf{R}-1, \mathbf{R}-2, \mathbf{R}-3 \text {, and } \\
& R-4 \text {, respectively } \\
& \bar{n}_{\mathrm{rc} 1}, \bar{n}_{\mathrm{rc} 2}, \bar{n}_{\mathrm{rc} 3}, \bar{n}_{\mathrm{rc} 4}=\text { Unit vectors in the direction }
\end{aligned}
$$


$S_{\mathrm{rc} 1}, S_{\mathrm{rc} 2}, S_{\mathrm{rc} 3}, S_{\mathrm{rc4}}=$ Shading factors on collectors due to reflectors $\mathrm{R}-1, \mathrm{R}-2$, $R-3$, and $R-4$, respectively

$s_{\mathrm{c}}, s_{\mathrm{r} 1}, s_{\mathrm{r} 2}, s_{\mathrm{r} 3}, s_{\mathrm{r} 4}=$ Tilts of the collector and reflectors R-1, R-2, R-3, and $R-4$ with respect to the horizontal, respectively $u_{1}, v_{1}, w_{1}=$ Components of the unit vector $\bar{n}_{\text {rcl }}$

$u_{2}, v_{2}, w_{2}=$ Components of the unit vector $\bar{n}_{\mathrm{rcl}}$

$u_{3}, v_{3}, w_{3}=$ Components of the unit vector $\bar{n}_{\mathrm{rcl}}$

$u_{4}, v_{4}, w_{4}=$ Components of the unit vector $\bar{n}_{\mathrm{rcl}}$

Greek letters

$$
\begin{aligned}
\beta= & \text { Solar altitude angle } \\
\gamma= & \text { Solar azimuth angle } \\
\delta= & \text { Solar declination } \\
\phi= & \text { Wall azimuth angle of the } \\
& \text { collector and reflectors } \\
& \text { measured east of south } \\
\rho= & \text { Reflectivity of the reflectors } \\
\theta_{\mathrm{c}}, \theta_{\mathrm{r} 1}, \theta_{\mathrm{r} 2}, \theta_{\mathrm{r} 3}, \theta_{\mathrm{r} 4}= & \text { Angles of incidence of beam } \\
& \text { radiation on the collector } \\
& \text { and reflectors R-1, R-2, R-3, } \\
& \text { and R-4, respectively }
\end{aligned}
$$

$\theta_{\mathrm{rc1}}, \theta_{\mathrm{rc} 2}, \theta_{\mathrm{rc} 3}, \theta_{\mathrm{rc} 4}=$ Angles of incidence for the reflected beam from reflectors $\mathbf{R}-1, \mathbf{R}-2, \mathbf{R}-3$ and R-4 and falling on the collector, respectively

$\tau \alpha=$ Transmittance-absorptance product

Subscripts

$\begin{aligned} b c= & \text { Beam radiation on the } \\ & \text { collector } \\ b r= & \text { Beam radiation on the }\end{aligned}$

$\mathrm{br}=\begin{aligned} & \text { Beam rad } \\ & \text { reflectors }\end{aligned}$

$c=$ Collector

cs $=$ Collector - sky

$\mathrm{DN}=$ Normal components of direct radiation

$\mathrm{dc}=$ Diffuse radiation on the collector

$\mathrm{r} 1, \mathrm{r} 2, \mathrm{r} 3, \mathrm{r} 4=$ Stand for reflectors $\mathrm{R}-1, \mathrm{R}-$ $2, \mathrm{R}-3$, and $\mathrm{R}-4$, respectively $\mathrm{rc} 1, \mathrm{rc} 2, \mathrm{rc} 3, \mathrm{rc} 4,=$ Rays reflected from reflectors $\mathrm{R}-1, \mathrm{R}-2, \mathrm{R}-3$, and $\mathrm{R}-4$ and falling on the collector, respectively 